\title{
Dystonic tics in patients with Gilles de la Tourette syndrome
}

\author{
Natalia Szejko ${ }^{1,2}$, Andrzej Jakubczyk ${ }^{3}$, Anna Dunalska ${ }^{2}$, Piotr Janik ${ }^{2}$ \\ ${ }^{1}$ Department of Bioethics, Medical University of Warsaw, Warsaw, Poland \\ ${ }^{2}$ Department of Neurology, Medical University of Warsaw, Warsaw, Poland \\ ${ }^{3}$ Department of Psychiatry, Medical University of Warsaw, Poland
}

\section{ABSTRACT}

Clinical rationale for the study. Gilles de la Tourette syndrome (GTS) is a childhood onset disorder characterised by motor and vocal tics. Different types of motor tics may occur in GTS, including dystonic tics (DTs). Although DTs have been recognised as part of GTS symptomatology, little is known about their risk factors or about how often and at what age they appear in affected individuals.

Aim of the study. The aim of our study was to investigate lifetime prevalence and clinical correlations of DTs in a Polish cohort of GTS patients.

Material and methods. We performed a prospective, one-registration study in a cohort of 207 consecutive ambulatory patients (mean age: $16.5 \pm 9.4$ years, 131 children, 162 males) with GTS. Duration of GTS was $9.0 \pm 8.0$ years (range: $1-39$ years). DTs were diagnosed during the interview. DTs were defined as slower and lasting longer than typical clonic tics, abnormal dystonia-like movements that led to a sustained, but not fixed, posture.

Results. DTs occurred at some point in the lifetime of $73.9 \%(n=153)$ of patients. The prevalence of DTs in adults and children was almost the same $(p=0.963)$. Age at onset of DTs was $9.9 \pm 5.2$ years with the most frequent onset in children ( $7-11$ years, $74.4 \%, n=64)$, followed by adolescence $(12-18$ years; $17.4 \%, n=15)$ and adulthood ( $\geq 18$ years, $8.1 \%, n=7)$. DTs occurred $3.7 \pm$ 4.2 years after tic onset. On average, patients suffered from $1.8 \pm 1.7$ types of DTs. The most frequent manifestations of DTs were: eyes (tightening resembling blepharospasm 84.3\%, $n=129$ and oculogyric crisis $45.8 \%, n=70$ ), trunk (dystonic postures 59.5\%, $\mathrm{n}=91$ ), jaw (bruxism 34.6\%, $\mathrm{n}=53)$, neck (30.7\%, $\mathrm{n}=47)$, upper limb $(26.1 \%, \mathrm{n}=40)$, and foot $(20.9 \%, n=32)$. Multivariate logistic regression analysis showed significant associations of DTs with the total number of simple, and the total number of complex, tics. Conclusions and clinical implications. DTs are early and frequent symptoms of GTS. They tend to localise in the facial area. DTs occur more frequently in individuals with a higher number of tics and probably add to the global impairment caused by tics.

Key words: Gilles de la Tourette syndrome, dystonic tics, simple tics, complex tics, dystonia

(Neurol Neurochir Pol 2019; 53 (5): 335-340)

\section{Introduction}

Gilles de la Tourette syndrome (GTS) is a childhood onset disorder characterised by motor and vocal tics. In up to $90 \%$ of patients, psychiatric comorbidities also occur. Different types of motor tics have been recognised as part of GTS symptomatology. Tics are generally brief. They can be categorised as clonic (less than $100 \mathrm{~ms}$ ) or dystonic and tonic (more than $300 \mathrm{~ms}$ ). Dystonic tics (DTs) are less common than clonic tics and are characterised by a repetitively abnormal posture similar to dystonia (e.g. torticollis). In tonic tics, there is a relatively long duration of contraction (in e.g. tension of abdominal muscles) but without exhibiting any abnormal postures.

DTs are known as a part of GTS symptomatology [1], but studies dedicated exclusively to DTs in GTS are scarce. Two reports describing this phenomenon were published by Jankovic and Stone in 1991 [2] and 1994 [3]. In the first study, 156 GTS patients were included and 57\% of them had DTs.

Address for correspondence: Piotr Janik, Department of Neurology, Medical University of Warsaw, Banacha 1a Srt., 02-091 Warsaw, Poland, e-mail: piotr.janik@wum.edu.pl 
The authors examined the patients not only clinically but in some cases, when DTs were present at the time of examination, they also used surface electromyography (EMG). The group was divided into two subgroups i.e. only clonic tics, and both clonic and dystonic tics. The patients with DTs had more head trauma, were suffering more frequently from attention deficit hyperactivity disorder (ADHD), and had more relatives who had tics. The next study dedicated to DTs was published by Jankovic in 1994 and focused on the treatment of DTs with botulin toxin. However, this study included just 10 patients, five with DTs that involved the eyes and five involving the neck muscles. This study focused merely on the effectiveness of botulin toxin.

A slightly different entity was described recently by Erro et al. [4]. They presented 11 patients with adult-onset primary DTs, which seems to be an intermediate disorder between tics and dystonia because it bears some characteristics of tics (localisation) and some of dystonia (sensory gestures, progression to dystonia in one case).

Finally, there have been two publications regarding co-existing tics and dystonia [5, 6]. Stone and Jankovic [5] described nine patients who developed dystonia additionally to their vocal and motor tics. Importantly, these patients were not treated with neuroleptics to exclude possible tardive dystonia. However, the authors underlined that the co-existence of both diseases could be mere chance due to the very small sample. Twenty years later, in 2011, Damásio et al. [6] analysed a group of 224 patients with tics; 20 of them developed dystonia. The group with dystonia was clearly distinguished from the patients without it, and the authors characterised the syndrome of a primary condition of tics associated with persistent focal/ segmental dystonia. The subjects with dystonia had later age of onset, lesser severity of tics, and lower frequency of associated features such as depression, obsessive compulsive symptoms (OCS) and ADHD.

\section{Clinical rationale for the study}

Although DTs have been recognised as part of GTS symptomatology, nonetheless little is still known about how often and at what age they appear in affected individuals, or if their occurrence is related to any clinical factors. Moreover, previous reports were only based on very small samples. Our study aimed to answer all of the above questions and confirm previous findings in a larger group of patients.

\section{Material and methods}

\section{Study participants}

Our cohort of GTS cases comprised 207 consecutive ambulatory patients aged 5-50 years (mean age: $16.5 \pm 9.4$ years; 162 males). The subjects were evaluated from 2013 to 2018. 131 children (62.6\%, mean age: $10.4 \pm 3.1$ years), and 76 adults (mean age: $27.2 \pm 7.4$ years) were enrolled. The mean age at tic onset was $6.4 \pm 2.7$ years. Duration of GTS was $4.9 \pm 3.0$ years (range: $0-13$ ) in children and $18.3 \pm 7.3$ years (range: $6-39$ ) in adults. $167(80.7 \%)$ patients had at least one psychiatric co-morbidity. To assess current tic severity, we used the Yale Global Tic Severity Scale (YGTSS) [7, 8]. The worst lifetime tic severity was investigated and each patient was asked to determine whether their peak tic severity was mild, moderate, or severe. The total number of tics was measured using the YGTSS: for each simple or complex vocal and motor tic, the patient received one point. The patients were evaluated for the clinical diagnosis of GTS and co-morbid mental disorders according to the Diagnostic and Statistical Manual of Mental Disorders (DSM-IV-TR). We questioned in order to determine different types of tics experienced by patients. OCS was diagnosed if obsessions and compulsions were egosyntonic, as opposed to egodystonic symptoms which characterise obssesive-compulsive disorder (OCD). The diagnosis of co-morbid mental disorders was also established on the basis of earlier psychiatric examinations that had been performed before the patient had been evaluated. This allowed us to address psychiatric disorders that are usually diagnosed in childhood (e.g. ADHD or oppositional defiant disorder) and also those for which symptoms were not yet present in adult patients at the time of examination. However, this applied only to a few subjects. We counted the total number of psychiatric disorders for each patient (defined according to DSM-IV-TR, the following disorders: ADHD, OCD, depression, anxiety disorder, conduct disorder, oppositional defiant disorder, pervasive developmental disorder, and learning disorder). Only the mental diseases classified in DSM were added to the total number of psychiatric disorders. All the patients were referred to neurologists experienced in movement disorders and were personally interviewed by the author of the study (PJ). The study was designed as a one-time registration study, and no new clinical data obtained on follow-up visits was included in the analysis.

The collection of clinical data from patients with GTS was approved by the Ethics Committee of the Medical University of Warsaw (KB/2/2007).

\section{Definition and differentiation of DTs}

DTs were defined as sustained contractions of muscles that led to abnormal, short-lasting, unfixed postures. We explained to the patients the differences between jerk-like movements (clonic tics) and slower and sustained DTs to facilitate the differentiation between those two movements. DTs were mainly differentiated into clonic tics, dystonia and tonic tics. As described by Jankovic [9], clonic tics are brief and jerk-like, involve only a single muscle or a group of muscles, and last less than $100 \mathrm{~ms}$. Tonic tics consist of isometric muscle tension, which is typically brief tension of abdominal or limb muscles that lasts more than $300 \mathrm{~ms}$. Finally, dystonia is abnormal posturing that ends in fixation, cannot be controlled voluntarily, and sometimes resolves after some gestures (gestes 
antagonistes). Patients and/or their caregivers were asked by the interviewer whether they had experienced such symptoms in the past or were experiencing them currently. We defined DTs as a current symptom when it had been present during the previous seven days, analogically to tic evaluation according to the YGTSS. In some cases, DTs were also witnessed at the time of examination or the diagnosis was supported by a video recording that had been provided by the subject or her/his family, or was recorded during the clinical evaluation. We additionally collected information about the anatomical region affected by DTs, and showed the patients examples of characteristic movements localised in each region (e.g. strong contraction of eyelid muscles resembling blepharospasm, slow head turning toward the arm similar to torticollis). As indicated by Ella et al. [10] and following Jankovic and Stone [2], we used the term 'bruxism' to describe DTs involving the mandibular region.

\section{Statistical analysis}

The statistical analyses were performed using STATISTICA ver 13.1 and SPSS ver 25 software. Normality of distribution was assessed using the Shapiro-Wilk test. For parametric variables, data was presented as arithmetic means and standard deviations (mean $\pm S D$ ). For non-parametric variables, data was presented as median and quartiles $(25 ; 75)$. Categorical variables were presented as frequencies (percentages). The parametric data was compared by an independent $t$-test and the nonparametric data by the Mann-Whitney U-test, as appropriate, and the categorical data was compared by the Fisher's exact test (two-sided).

In both analyses, comparisons between groups were considered significantly different when a two-tailed test was $\mathrm{p}<$ 0.05 . All variables that were significant in the primary analyses were entered into a logistic regression analysis in order to determine risk factors for DTs in GTS patients. In addition, gender and age were entered into the multivariate model as control variables.

\section{Results}

DTs occurred at some point in the lifetime of $73.9 \%$ (n $=153$ ) of patients, in 57 adults and 96 children. In $41.2 \%$ of patients $(n=63)$, DTs had only been present in the past and not at the time of evaluation. The prevalence of DTs in adults and children was almost the same $(\mathrm{p}=0.963,75 \%, \mathrm{n}=57 \mathrm{vs}$. $73.2 \%, \mathrm{n}=96$, respectively). Age at onset of DTs was known only in $57 \%(\mathrm{n}=86)$ of patients, with the most frequent onset in children ( $7-11$ years, $74.4 \%, \mathrm{n}=64)$, followed by adolescence $(12-18$ years; $17.4 \%, \mathrm{n}=15)$ and adulthood $(\geq 18$ years, $8.1 \%, \mathrm{n}=7)$. The age of onset was known in 23 adults $(23 / 57$, $40.4 \%)$ and in 63 children $(63 / 96,65.6 \%)$. Mean age of DTs onset was $9.9 \pm 5.2$ years compared to $6.4 \pm 2.8$ years for GTS onset. On average, patients suffered from $1.8 \pm 1.7$ types of DTs. The most frequent localisations of DTs were: eyes (tightening resembling blepharospasm $84.3 \%, \mathrm{n}=129$ and oculogyric crisis $45.8 \%, \mathrm{n}=70$ ), trunk (dystonic postures $59.5 \%, \mathrm{n}=91$ ), jaw (bruxism 34.6\%, n = 53), neck $(30.7 \%, n=47)$, upper limb $(26.1 \%, \mathrm{n}=40)$, and foot $(20.9 \%, \mathrm{n}=32)$.

In univariate analyses, patients with DTs suffered from more severe tics, had more simple and complex tics, and more psychiatric comorbidities (ADHD, OCD, depression, anxiety disorder, conduct disorder, pervasive developmental disorder, oppositional defiant disorder, and learning disorder). Multivariate logistic regression analysis showed only significant associations of DTs with total number of simple and total number of complex tics. The results of univariate und multivariate analysis are shown in Table 1.

\section{Discussion}

Our study is one of the very few to have reported about DTs in GTS. Previous reports date back to the 1990s and include smaller study samples. In our sample, DTs were found in the majority of patients (73.9\%), they started mainly in childhood and adolescence, were located in the face, and correlated with the total number of tics.

In the publication by Jankovic and Stone from 1991 [2], the prevalence of DTs was lower than in our group. This could be related to a different methodology. Jankovic and Stone used additionally EMG in some cases to determine the exact duration of the tic. Clonic tics last less than $100 \mathrm{~ms}$ while dystonic last more than $300 \mathrm{~ms}$. However, they used this measurement only in some patients and did not report whether the amount of patients with DTs had changed after using surface EMG. We can only suspect that the amount of patients categorised as DTs+ in our sample could be liable to change if one were to apply surface EMG. Moreover, Jankovic and Stone used DSM III criteria to diagnose GTS and their sample was smaller than ours (156 vs. 207). In the study by Jankovic and Stone the patients with DTs had more ADHD and head trauma. In our sample, univariate analysis showed that patients with DTs were more affected by tics as well as having more psychiatric comorbidities, but multivariate regression confirmed only a relationship with the number of motor and vocal tics.

The age at onset was known in only $57 \%$ of patients. Unsurprisingly, it was better known in children $(65.6 \%)$ than in adults (40.4\%). This could be related to the fact that usually DTs start in childhood or adolescence and that therefore some adults could not recall when DTs had started due to the long interval between the onset of DTs and the clinical evaluation. Additionally, in children with DTs, the medical history was always supported by information given by at least one parent.

Although we did not use objective measurements to assess impairment due to tics, we believe that DTs may add to global impairment, which was confirmed by correlating with the YGTSS. We speculate that also a correlation between the total number of tics and lifetime peak tic severity indicates that such patients have more tics and, in consequence, this can also 
Table 1. Characteristics and associations of DTs

\begin{tabular}{|c|c|c|c|}
\hline \multicolumn{4}{|c|}{ General comparison between DTs+ and DTs- groups } \\
\hline \multirow{2}{*}{ Variable } & \multicolumn{3}{|c|}{$\begin{array}{l}\text { GTS patients } \\
\quad(n=203)\end{array}$} \\
\hline & $\begin{array}{l}\text { Patients with DTs } \\
\quad(n=153)\end{array}$ & $\begin{array}{l}\text { Patients without DTs } \\
\qquad(\mathrm{n}=54)\end{array}$ & $\mathbf{p}$ \\
\hline $\begin{array}{l}\text { Age at evaluation [years] } \\
\text { [median] (IQR) }\end{array}$ & $14(9-22)$ & $11(9-23)$ & 0.60 \\
\hline Gender (male/female) & $124 / 29$ & $38 / 16$ & 0.102 \\
\hline YGTSS [median] (IQR) & $50(31-65)$ & $35(21-49)$ & 0.0004 \\
\hline Total number of simple tics [mean \pm SD] & $13.73 \pm 4.76$ & $9.36 \pm 3.92$ & 0.000 \\
\hline Total number of complex tics [median] (IQR) & $8(5-13)$ & $3(1-6)$ & 0.000 \\
\hline Number of mental disorders [median] (IQR) & $2(1-3)$ & $1(0-2)$ & 0.002 \\
\hline Medication for tics [at evaluation] & $\mathrm{n}=67(43.8 \%)$ & $n=21(38.9 \%)$ & 0.531 \\
\hline Peak tic severity [median] (IQR) & $2(2-3)$ & $2(1-2)$ & 0.000 \\
\hline Family history of tics or GTS & $\mathrm{n}=35(22.9 \%)$ & $n=13(24.1 \%)$ & 0.934 \\
\hline Family history of OCD or OCS & $\mathrm{n}=52(34.0 \%)$ & $\mathrm{n}=15(27.8 \%)$ & 0.276 \\
\hline \multicolumn{4}{|c|}{ Logistic regression model } \\
\hline & & $\begin{array}{c}\text { Multivariate } \\
\text { Analysis }\end{array}$ & \\
\hline Variable & \multicolumn{2}{|c|}{ OR $(95 \% \mathrm{Cl})$} & $\mathbf{p}$ \\
\hline Age & \multicolumn{2}{|c|}{$1.019(0.972-1.068)$} & 0.429 \\
\hline Gender & \multicolumn{2}{|c|}{$0.755(0.315-1.813)$} & 0.529 \\
\hline Peak tic severity & \multicolumn{2}{|c|}{$1.509(0.716-3.180)$} & 0.279 \\
\hline YGTSS & \multicolumn{2}{|c|}{$0.974(0.907-1.046)$} & 0.472 \\
\hline Total number of simple tics & \multicolumn{2}{|c|}{$1.174(1.042-1.323)$} & 0.008 \\
\hline Total number of complex tics & \multicolumn{2}{|c|}{$1.248(1.082-1.440)$} & 0.002 \\
\hline Number of mental disorders & \multicolumn{2}{|c|}{$0.854(0.629-1.160)$} & 0.312 \\
\hline
\end{tabular}

$\mathrm{n}$ - number of patients; IQR - interquartile range; SD — standard deviation; OR — odds ratio; Cl — confidence interval; DTs — dystonic tics; GTS - Gilles de la Tourette Syndrome; YGTSS - Yale Global Tic Severity Scale; OCD — obsessive-compulsive disorder; OCS - obsessive-compulsive symptoms; $\mathrm{p}$ - DTs + vs. DTs-; $\mathrm{p}$ value $<0.05$ are shown in bold. For non-parametric variables, data is presented as median and interquartile range. Categorical variables are presented as frequencies (percentages)

aggravate global impairment in some patients. Nevertheless, patients with DTs did not receive more frequently medication for tics.

Similarly to other studies, also in our group, DTs tended to localise in the head and neck area. This localisation is also typical for clonic tics. Interestingly, in a group of patients with co-existing dystonia and tics described by Damásio et al., the most frequent was cervical dystonia [6].

DTs must be differentiated from primary dystonia. The main difference is that while DTs are characterised by a temporarily sustained posture, in dystonia the abnormal posturing is fixed. Also, the dystonia is constant, with a fixed pattern of movement, while DTs wax and wane in intensity and localisation, as do all tics in GTS. Helpful clues based on clinical observations that could help to differentiate DTs from dystonia are summarised in Table 2.
Table 2. Distinguishing features between dystonic tics and dystonia

\begin{tabular}{lcc}
\multicolumn{1}{c}{ Parameter } & DTs & Dystonia \\
Male predominance & + & - \\
Co-occurrence of phonic and clonic tics & + & - \\
Suppressibility & + & - \\
Growing inner tension during suppression & + & - \\
Premonitory urge & + & - \\
Waxing and waning course & + & - \\
Amelioration in adulthood & + & - \\
Geste antagoniste & - & + \\
Fixed posture & - & + \\
Unchanged pattern & - & +
\end{tabular}

DTs - dystonic tics 
None of the patients in our sample had a history of dystonia. Nevertheless, Damásio et al. [6] detected 20 cases of dystonia $(8.9 \%)$ in his sample of 224 patients with tics. The group of patients included in this study was very heterogenous, some of the patients had tic onset in childhood, some in adulthood, plus patients with GTS but also chronic tic disorder were enrolled. This could possibly bias the results. Previously, Stone and Jankovic [5] presented nine cases (5\%) of GTS and dystonia. It seems to be, therefore, a quite rare phenomenon, but one that should be taken into consideration. Nevertheless, the authors mention that those numbers could be biased as only severely affected patients are treated in Movement Disorders Centers, where their study was conducted.

The second disorder that can resemble DTs is tardive dyskinesia, especially tardive dystonia. Tardive dyskinesia is a disorder characterised by involuntary movements, typically of the orofacial muscles and also of the extremities and other muscle groups. The condition is associated with exposure to dopamine receptor blocking agents, including antipsychotics that are commonly used in the treatment of tics. Among psychiatric patients, the risk of developing tardive dyskinesia is quite high, especially when treated with first generation antipsychotics and when this treatment is long-lasting. Correll et al. reported the annual prevalence of tardive dyskinesia as being as high as $3.9 \%$ for first generation antipsychotics and $5.5 \%$ for second generation antipsychotics [11]. Müller-Vahl and Krüger [12] analysed records of 521 GTS patients treated with neuroleptics. None of them developed tardive dyskinesia, but most patients were treated with neuroleptics for less than a year. Although tardive dyskinesia seems to be a quite rare side effect in GTS, it should be considered. To distinguish tardive dystonia from DTs we should consider the exposure to neuroleptics. Tardive dystonia usually persists for longer, even after discontinuation of the offending drug. DTs are tics, so their intensity changes, and they can even disappear suddenly. DTs are not permanent, but there are periods of remission and relapse. Furthermore, slow tapering should improve, while sudden dose reduction may aggravate, drug-induced dystonia.

The next type of tics that should be taken into consideration in a differential diagnosis of DTs are tonic tics. Similarly to DTs, tonic tics are slow, but they do not lead to sustained posturing. Typical tonic tics are located in the abdominal or limb muscles. It is often unclear whether the subject is suffering from DTs or tonic tics, and it is crucial to explain the difference to the patient. However, we did not find any correlation between DTs and tonic tics ( $\mathrm{p}=0.103)$.

Finally, Erro et al. [4] suggested that patients who develop DTs during adulthood represent a totally different group which they named adult-onset primary dystonic tics. From their database of 253 , they found 21 with DTs and 10 of them developed DTs after the age of 18 as the first manifestation of an unspecified tic disorder. Some of those movements were evaluated posteriorly to dystonia, so the authors suggested that adult onset primary dystonic tics represent a transient entity between tics and dystonia. In our sample, only seven patients had DTs onset in adulthood $(7 / 153,4.6 \%)$. To the best of our knowledge, none of them developed dystonia or had other features that resembled dystonia such as geste antagoniste.

\section{Clinical implications/future directions}

DTs are frequent and early symptoms in GTS and should be considered by clinicians. Therefore, we believe that our study has an important clinical value. DTs normally appear in the facial region. DTs occur in patients who have more tics and they probably aggravate the impairment caused by tics. They should be differentiated from primary focal/segmental dystonia, tonic tics and tardive dystonia. We should therefore take into account that patients with DTs may require more clinical attention due to greater impairment. As indicated by Jankovic [3], botulin toxin could also be used in the treatment of DTs.

\section{Limitations}

The biggest limitation of our study is the recall bias. Many adult patients did not remember the presence or the age of onset of DTs in their childhood, and only in some cases was it possible to obtain this additional information from a family member. We also did not use superficial EMG to make the assessment more objective. Another objective method, video recording, was available only in some cases in our study. The design of our one-time registration study may have also influenced the prevalence of DTs. Moreover, we used different methods of data collection from children as opposed to adults. Most clinical information regarding children was provided by their parents, whereas adults reported themselves.

\section{Funding: This publication was prepared without any external source of funding.} Conflicts of interest: None to declare.

\section{References}

1. Cath DC, Hedderly T, Ludolph AG, et al. ESSTS Guidelines Group. European clinical guidelines for Tourette syndrome and other tic disorders. Part I: assessment. Eur Child Adolesc Psychiatry. 2011; 20(4): 155-171, doi: 10.1007/s00787-011-0164-6, indexed in Pubmed: 21445723.

2. Jankovic J, Stone L. Dystonic tics in patients with Tourette's syndrome. Mov Disord. 1991; 6(3): 248-252, doi: 10.1002/mds.870060309, indexed in Pubmed: 1922130.

3. Jankovic J. Botulinum toxin in the treatment of dystonic tics. Mov Disord. 1994; 9(3): 347-349, doi: 10.1002/mds.870090315, indexed in Pubmed: 8041378.

4. Erro R, Martino D, Ganos C, et al. Adult-Onset Primary Dystonic Tics: A Different Entity? Mov Disord Clin Pract. 2014; 1(1): 62-66, doi: 10.1002/mdc3.12005, indexed in Pubmed: 30363833. 
5. Stone LA, Jankovic J. The coexistence of tics and dystonia. Arch Neurol. 1991; 48(8): 862-865, doi: 10.1001/archneur.1991.00530200104028, indexed in Pubmed: 1898264.

6. Damásio J, Edwards MJ, Alonso-Canovas A, et al. The clinical syndrome of primary tic disorder associated with dystonia: a large clinical series and a review of the literature. Mov Disord. 2011; 26(4): 679-684, doi: 10.1002/mds.23484, indexed in Pubmed: 21506147.

7. Leckman JF, Riddle MA, Hardin MT, et al. The Yale Global Tic Severity Scale: initial testing of a clinician-rated scale of tic severity. J Am Acad Child Adolesc Psychiatry. 1989; 28(4): 566-573, doi: 10.1097/00004583198907000-00015, indexed in Pubmed: 2768151.

8. Stefanoff P, Wolańczyk T. [Validity and reliability of Polish adaptation of Yale Global Tic Severity Scale (YGTSS) in a study of Warsaw schoolchil- dren aged 12-15]. Przegl Epidemiol. 2005; 59(3): 753-762, indexed in Pubmed: 16433318.

9. Jankovic J. Phenomenology and classification of tics. Neurologic Clinics. 1997; 15(2): 267-275, doi: 10.1016/s0733-8619(05)70311-x.

10. Ella B, Ghorayeb I, Burbaud P, et al. Bruxism in Movement Disorders: A Comprehensive Review. J Prosthodont. 2017; 26(7): 599-605, doi: 10.1111/jopr.12479, indexed in Pubmed: 27077925.

11. Correll CU, Schenk EM. Tardive dyskinesia and new antipsychotics. Curr Opin Psychiatry. 2008; 21(2): 151-156, doi: 10.1097/YC0.0b013e3282f53132, indexed in Pubmed: 18332662.

12. Müller-Vahl KR, Krueger D. Does Tourette syndrome prevent tardive dyskinesia? Mov Disord. 2011; 26(13): 2442-2443, doi: 10.1002/ mds.23894, indexed in Pubmed: 21956454. 